\title{
Interaction of Heliothis armigera Nuclear Polyhedrosis Viral Capsid Protein with its Host Actin
}

\author{
Song-Ya Lu, Yi-Peng Qi* and Guo-Qiong Ge \\ Institute of Virology, Wuhan University, Wuhan 430072, P.R. China
}

Received 29 July 2002, Accepted 20 August 2002

In order to find the cellular interaction factors of the Heliothis armigera nuclear polyhedrosis virus capsid protein VP39, a Heliothis armigera cell cDNA library was constructed. Then VP39 was used as bait. The host actin gene was isolated from the cDNA library with the yeast two-hybrid system. This demonstrated that VP39 could interact with its host actin in yeast. In order to corroborate this interaction in vivo, the vp39 gene was fused with the green fluorescent protein gene in plasmid pEGFP39. The fusion protein was expressed in the Hz-AM1 cells under the control of the Autographa californica multiple nucleopolyhedrovirus immediate early gene promoter. The host actin was labeled specifically by the red fluorescence substance, tetramethy rhodamine isothicyanete-phalloidin. Observation under a fluorescence microscopy showed that VP39, which was indicated by green fluorescence, began to appear in the cells $6 \mathrm{~h}$ after being transfected with pEGFP39. Red actin cables were also formed in the cytoplasm at the same time. Actin was aggregated in the nucleus $9 \mathrm{~h}$ after the transfection. The green and red fluorescence always appeared in the same location of the cells, which demonstrated that VP39 could combine with the host actin. Such a combination would result in the actin skeleton rearrangement.

Keywords: Actin, Capsid protein VP39, Green fluorescent protein, Heliothis armigera nuclear polyhedrosis virus, Yeast two-hybrid system

\section{Introduction}

Heliothis armigera is the most dangerous cotton pest in P.R. China, which causes significant damage to corn, tobacco, tomato, and wheat. Many years of chemical control to the pest

*To whom correspondence should be addressed.

Tel: 86-27-87682938; Fax: 86-27-87661831

E-mail: qiyipeng@whu.edu.cn have led to its serious resistance and environmental pollution. The Heliothis armigera nuclear polyhedrosis virus (HaNPV), due to its specifity and harmlessness to other organisms as well as the environment, has been used as a commercial bioinsecticide for years. But compared to chemicals, HaNPV kills pests slower, which decreases its effect and limits its wide application (Peng et al., 1998). We are now researching the infection mechanism of HaNPV and hope to improve its pest-killing effect.

Like the Autographa californica multiple nuclear polyhedrosis viruses (AcMNPV), the type-species of Nuclepolyhedrovirus genus, larvae infected by HaNPV also results in the liquidization of the host. It was discovered that the AcMNPV capsid protein P39 is related to the rearrangement and polymerization of host actin (Chariton et al., 1991; 1993). The conformation-changed actin is more suitable for proteolysis by another viral protease, V-CATH, and leads to the host liquidization (Lorene et al., 1996). In addition, AcMNPV VP39 is needed in the host nuclear actin polymerization that is required for AcMNPV nucleocapsid morphogenesis (Taro et al., 1999).

The vp39 gene of AcMNPV, the major component of the capsid, is $1044 \mathrm{bp}$. The vp39 gene of the Heliothis armigera nuclear polyhedrosis virus (HaNPV) that we isolated and identified is $882 \mathrm{bp}$. It has a $54 \%$ and $40 \%$ homology with that of AcMNPV in the DNA and amino acid sequences, respectively (Lorene et al., 1996; Chen et al., 2001). The objective of the current study is to corroborate the function of the HaNPV capsid protein VP39 in virus infection.

\section{Materials and Methods}

Insect cell culture, bacterial strain, yeast cell, and plasmids Hz-AM1 cells, provided by Dr. Liu Deli (Huazhong Normal University, P.R. China), were the host for the VP39 transient expression. The cells were cultured in Grance's insect cell culture medium that was supplemented with $10 \%$ fetal bovine serum (FBS) at $28^{\circ} \mathrm{C}$. E. coli $\mathrm{DH} 5 \alpha$, originating from our laboratory, was host for all of the bacterial plasmid manipulations. The yeast two-hybrid 


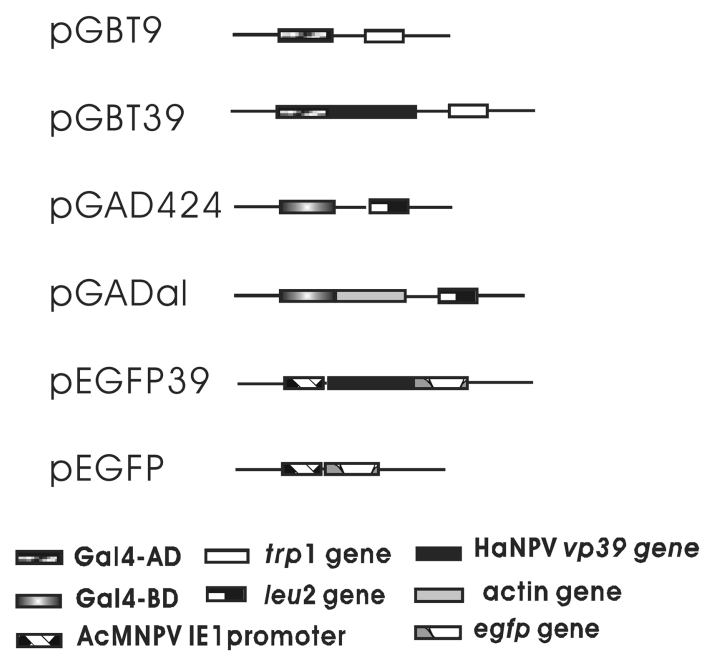

Fig. 1. A map of all of the vectors that were used and constructed in this paper.

system was purchased from Clontech (Franklin Lakes, USA). Yeast HF7C, which was grown in YPD medium at $30^{\circ} \mathrm{C}$, was the host for pGBT9, pGBT39, pGAD424, pGADal, the Ha-cDNA library plasmid, and $\beta$-galactosidase positive control plasmid pCL1. The plasmid pGBT9 contains a trpl gene and Gal4 DNA-binding domain (Gal4-BD), the yeast transformants of which can be grown on a SD/-Trp plate. The plasmid pGAD424 contains a leu2 gene and Gal4 activation domain (Gal4-AD), the yeast transformants of which can be grown on a SD/-Leu plate. Figure 1 shows a map of all of the vectors that were used and constructed in this paper.

Chemicals and buffers Yeast media YPD, SD/-Trp, SD/-TrpLeu, and SD/-Trp-Leu-His were purchased from Clontech. The TRIZOL reagent, Oligo (dT) cellulose columns, SuperScript ${ }^{\mathrm{TM}}$ Choice System for cDNA Synthesis kit, Grace's insect cell culture medium, FBS, and LipofectAMINE ${ }^{\mathrm{TM}} 2000$ reagent were purchased from Invitrogen Co. (New York, USA). TRITC-phalloidin was purchased from Sigma Chemical Co. (St. Louis, USA). T4 DNA ligase, restriction endonucleases, and other common chemicals were obtained from Hua-Mei Co. (Wuhan, P. R. China). The Z buffer/X-gal solution $\left[100 \mathrm{ml} \mathrm{Z}\right.$ buffer $\left(16.1 \mathrm{~g} / 1 \mathrm{Na}_{2} \mathrm{HPO}_{4} \cdot 7 \mathrm{H}_{2} \mathrm{O}\right.$, $5.50 \mathrm{~g} / \mathrm{l} \mathrm{NaH} \mathrm{PO}_{4} \cdot \mathrm{H}_{2} \mathrm{O}, 0.75 \mathrm{~g} / \mathrm{l} \mathrm{KCl}, 0.246 \mathrm{~g} / \mathrm{l} \mathrm{MgSO}{ }_{4} \cdot 7 \mathrm{H}_{2} \mathrm{O}, \mathrm{pH}$ 7.0), $0.27 \mathrm{ml} \beta$-mercaptoethanol, and $1.67 \mathrm{ml} \mathrm{X}$-gal stock solution $(20 \mathrm{mg} / \mathrm{ml} \mathrm{X}$-gal in N,N-dimethylformamide)], PHEM buffer [60 mM piperazine-N,N'-bis (2-ethanesulfonic acid), 25 mM N'-2hydroxyethylpiperazine-N-2-ethanesulfonic acid, $10 \mathrm{mM}$ EGTA, and $2 \mathrm{mM} \mathrm{MgCl}_{2}(\mathrm{pH} 6.9$ with $\mathrm{NaOH})$ ], as well as other buffers were prepared in our laboratory.

Ha-cDNA library construction Using the oligo (dT) cellulose column, mRNA of the Hz-AM1 cells was purified from the total RNA that was isolated from the Hz-AM1 cells with the TRIZOL reagent. The cDNA of the Hz-AM1 cells (Ha-cDNA) was synthesized with the SuperScript ${ }^{\mathrm{TM}}$ Choice System from a cDNA Synthesis kit. Inserting Ha-cDNA with the EcoRI adapter into the EcoRI site of pGAD424 resulted in the construction of Ha-cDNA library plasmids. Transforming E. coli DH5 $\alpha$ with the library plasmids allowed the construction of the Ha-cDNA library.

Yeast two-hybrid system The yeast two-hybrid system was manipulated according to the Clontech Yeast Protocols Handbook. First, the open-reading frame of the HaNPV vp39 gene was fused in frame with the Gal4 DNA-binding domain in the vector pGBT9, which resulted in the bait plasmid pGBT39. Then, according to the library-scaled sequential transformation protocol of the yeast twohybrid system, pGBT39 was used to transform the yeast HF7C cells that originally carried the Ha-cDNA library plasmids. Next, the transformation mixture was plated onto the SD/-Trp-Leu-His plates and all of the Trp+Leu+His+ clones were streaked onto fresh $\mathrm{SD} /$-Trp-Leu-His plates for $\beta$-galactosidase filter assay after $4 \mathrm{~d}$. Finally, the His+Lac+ clones were identified by sequencing.

Cell transfection The HaNPV vp39 transient expression vector pEGFP39 was constructed by inserting vp39 in frame with the green fluorescent protein (GFP) gene under the control of the immediate early (IE1) gene promoter of AcMNPV. The negativecontrol plasmid pEGFP only contained the $g f p$ gene under the control of the AcMNPV IE1 promoter. Using the LipofectAMINE ${ }^{\mathrm{TM}} 2000$ reagent, the Hz-AM1 cells that were grown on coverslips in 6-well plates were transfected with pEGFP39 in order to express the fusion protein GFP-VP39. The cells that were transfected with pEGFP were used to express GFP.

Fluorescence microscopy At 6, 9, 12, and $24 \mathrm{~h}$ after being transfected with pEGFP39 or pEGFP, the cells on the coverslips were extracted for $10 \mathrm{~min}$ in a PHEM buffer that contained $0.15 \%$ Triton X-100 and $3 \times 10^{-7} \mathrm{M}$ TRITC-phalloidin. TRITC-phalloidin was able to bind to the actin protein specifically and emit red fluorescence under fluorescence microscopy. After being rinsed twice with PHEM buffer, the extracted cells were mounted for viewing in a drop of a nonbleach mountant (10 mg of phenylenediamine/ml of PHEM mixed 19 with glycerol). Then the coverslips were viewed and photographed with a photomicroscope that was equipped for fluorescence microscopy

\section{Results}

Quantity of the Ha-cDNA library The titer of the HacDNA library was $2.1 \times 10^{6} \mathrm{cfu} / \mathrm{ml}$ calculated by spreading diluted-library transformants onto LB/amp plates, then counting the number of colonies that were grown on the plates. The Ha-cDNA insert size of 10 randomly-selected clones was determined by PCR amplification using insertscreening primers. Fig. 2 shows that 7 of them had different inserts ranging from about $0.6-9 \mathrm{~kb}$. Therefore, the independent clones of the library were $\left(2.1 \times 10^{6}\right) \times 0.7=$ $1.5 \times 10^{6} \mathrm{cfu} / \mathrm{ml}$. The Ha-cDNA library could represent the mRNA population complexity of the Hz-AM1 cells.

Interaction of VP39 with actin in yeast We used the yeast two-hybrid system to screen the cellular interaction protein of HaNPV VP39 from the Ha-cDNA library. The open-reading frame of the $v p 39$ gene that was fused in frame with the Gal4 DNA-binding domain in the pGBT9 plasmid was used as bait. 


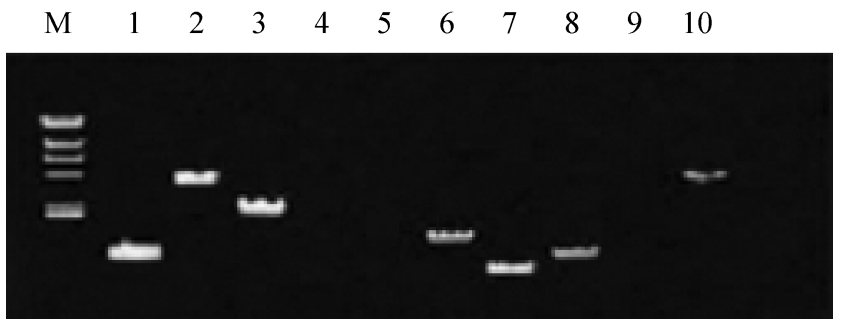

Fig. 2. PCR analysis of the cDNA insert in the Ha-cDNA library plasmids. Lane M, DNA size marker (from top to bottom: $23.1 \mathrm{~kb}, 9.4 \mathrm{~kb}, 6.6 \mathrm{~kb}, 4.4 \mathrm{~kb}, 2.3 \mathrm{~kb}$, and $2.0 \mathrm{~kb}$ ); Lanes 1-10, inserts of the cDNA in the Ha-cDNA library plasmids amplified by PCR.

From $1 \times 10^{6}$ co-transformants, one His+LacZ+ clone was isolated. The full-length of the inserted cDNA was 1298bp (Fig. 3). It encoded a polypeptide of 376 amino acids, which was just the same as the actin of Helicoverpa armigera (Rourke et al., 1997).

To conform the interaction of HaNPV VP39 actin protein, the open-reading frame of the actin gene was fused in frame to the Gal4-activation domain in vector pGAD424, which resulted in the Gal4-AD/actin fusion protein expression vector pGADal (Fig. 1). As shown in Figure 4, the HF7C yeast co-

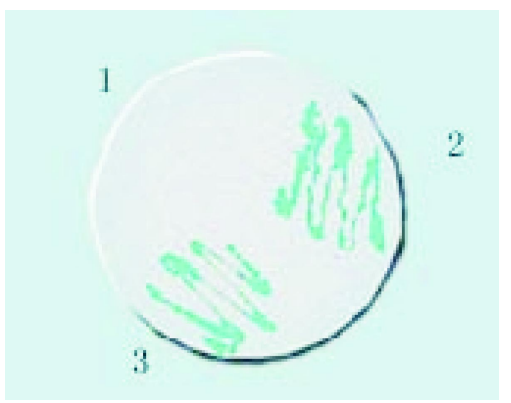

Fig. 4. $\beta$-galactosidase filter assay for interaction of VP39 with host actin in yeast. The yeast transformants that were grown on the SD/-Trp-Leu-His plate at $30^{\circ} \mathrm{C}$ for $3 \mathrm{~d}$ were clung to a sterile VWR grade 410 filter, and then submerged in a pool of liquid nitrogen for $10 \mathrm{~s}$. The filter was then placed on another filter that had been presoaked with a Z-buffer/X-gal solution to produce blue colonies. Top left 1 , the negative-control yeast that contained pGBT39 and pGAD424; Top right 2, the yeast that contained the positive-plasmid pCL1; Bottom 3, the yeast that contained pGBT39 and pGADal.

transformant of plasmids pGADal and pGBT39 grew very well on the SD/-Trp-Leu-His plate, and displayed a strong blue color in the $\beta$-galactosidase filter assay. However, the cotransformant of pGADal and pGBT9 could not grow on the

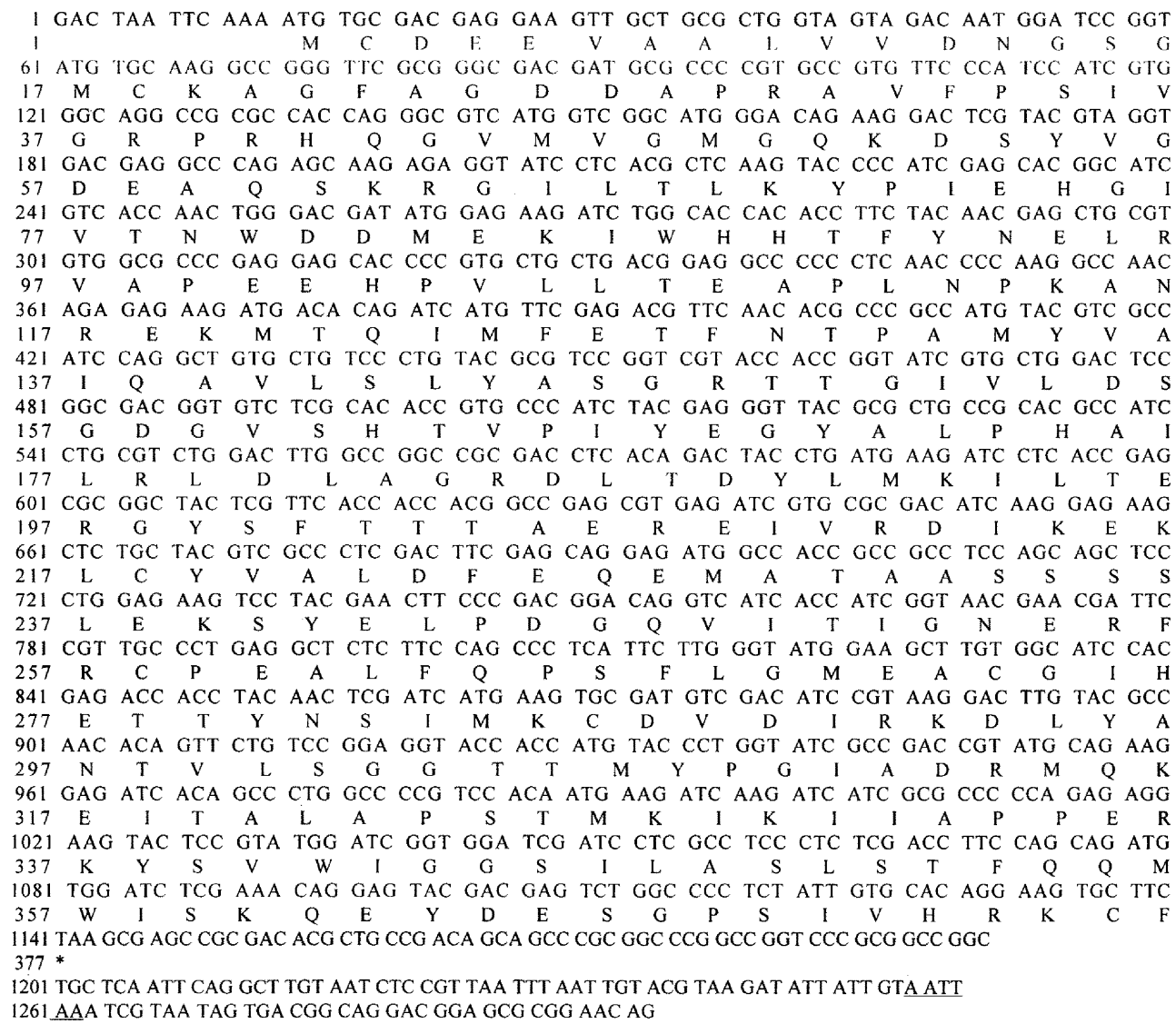

Fig. 3. DNA sequence and deduced amino acids of the isolated actin gene. AATTAA is the poly (A) signal. 

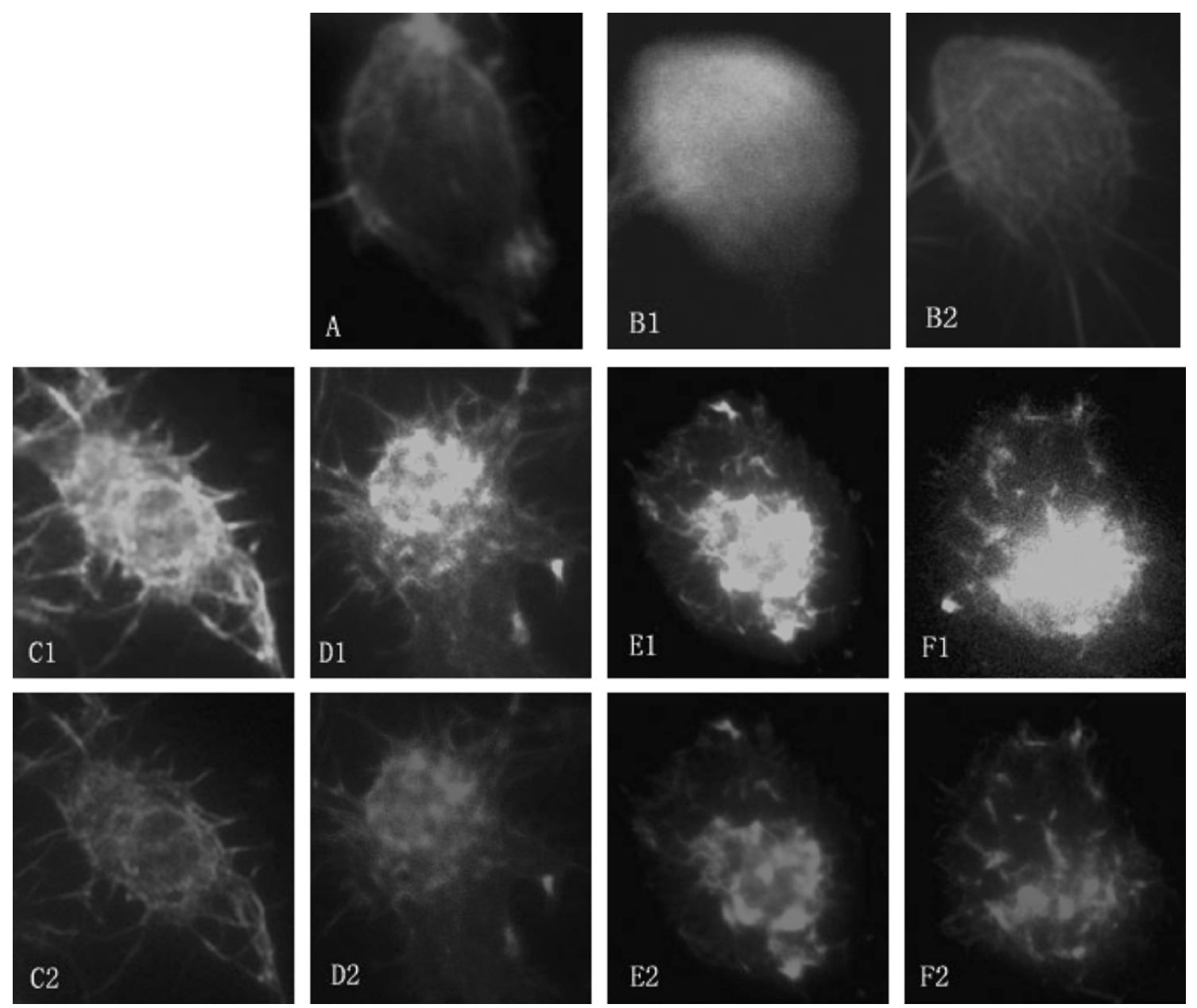

Fig. 5. Transient expression of HaNPV VP39 and the actin skeleton in the Hz-AM1 cells observed under fluorescence microscopy. The actin skeleton in the Hz-AM1 cells was stained specifically by the red fluorescent substance TRITC-phalloidin. GFP and the fusion protein GFP-VP39 that was expressed in the Hz-AM1 cells were shown by the green fluorescence that was emitted by the green fluorescent protein. A, the actin skeleton in the normal Hz-AM1 cell; B1, the GFP that was expressed in the Hz-AM1 cell that was transformed with pEGFP; B2, the actin skeleton in the same cell of B1; C1, D1, and E1, the fusion protein GFP-VP39 that was expressed transiently in the Hz-AM1 cells 6, 9, 12, and $24 \mathrm{~h}$ after being transfected with pEGFP39; C2, D2, and E2, the actin skeleton in the corresponded cells of $\mathrm{C} 1, \mathrm{D} 1$, and $\mathrm{E} 1$.

same plate. These results demonstrate that two fusion proteins, Gal4-BD/VP39 and Gal4-AD/ACTIN that were expressed in the yeast HF7C that contained plasmids pGBT39 and pGADal, can form a transcription-activation factor because of the interaction between VP39 and actin. These proteins also activate the his and lac Z gene expressions under the control of the Gal4-responsive promoter. Therefore, the HF7C co-transformants that displayed the His+LacZ+ phenotype confirmed the interaction of HaNPV VP39 with its host actin in yeast.

Actin rearrangement in $\mathrm{Hz}-\mathrm{AM1}$ cells induced by the transient expression of HaNPV VP39 The GFP expression or the fusion protein VP39-GFP in cells was observed under fluorescence microscopy. No green fluorescence was discovered in the pEGFP or pEGFP39
transfected-Hz-AM1 cells before $6 \mathrm{~h}$ after the transfection, which indicates that the GFP protein or GFP-VP39 was not expressed before that time. The actin skeleton in these cells, which was illuminated by a red fluorescence substance TRITC-phalloidin, was just like that of the normal cells (data not shown).

Six hours after the transfection, the fusion protein GFPVP39 began to appear in the cell plasma (Fig. 5C1), and the actin skeleton began to rearrange itself (Fig. 5C2). After $9 \mathrm{~h}$, the actin skeleton was further aggregated in the cytoplasm and began to appear in the nucleus. The cells then became round (Fig. 5D2). At $12 \mathrm{~h}$ and $24 \mathrm{~h}$ after the transfection, the actin skeleton formed thick aggregates in the nucleus (Fig. 5E2, F2).

On the other hand, the Hz-AM1 cells that were transfected with pEGFP that contained the $g f p$ gene under the control of 
the AcMNPV IE1 promoter could express the green from 6$24 \mathrm{~h}$ after the transfection (Fig. 5B1). But, the host actin skeleton in the cell did not change (Fig. 5B2), it remained just like those of normal cells (Fig.5A). This result indicates that the expressed GFP could not affect the host actin skeleton.

As shown in Figure 5, the location and density of the green fluorescence in the pEGFP39-harbored cells corresponded to the red fluorescence ( $\mathrm{C} 1$ corresponded to $\mathrm{C} 2, \mathrm{D} 1$ to $\mathrm{D} 2, \mathrm{E} 1$ to $\mathrm{E} 2$, and F1 to F2 in Fig. 5). But, there was no such correspondence in the pEGFP-harbored cells (B1 did not correspond to B2 in Fig. 5). These results suggest that HaNPV VP39 could bind actin and cause the rearrangement of the host actin skeleton, but GFP had no such function.

\section{Discussion}

Actin is an abundant and highly-conserved protein in eukaryotic cells, and is the major component of the cell skeleton. Actin may be involved in normal cellular processes, such as adhesion, motility, division, phagacytosis, secretion, and the intracellular transport of organelles (Bradley et al., 1979; Kachar et al., 1988; Stockem et al., 1983; Stossel, 1984; Tasaka et al., 1988). Actin in particular plays an important role in the infectious course of the virus.

VP39 and V-CATH are the most important proteins that are associated with the host liquidization. AcMNPV VP39 could induce the rearrangement of the actin cytoskeleton. The rearranging actin cytoskeleton is more suitable for the viral protease V-CATH to degrade (Lorene et al., 1996). Larvae that infected by AcMNPV with two copies of $v$-cath achieved liquidization easier and died more quickly than those that were infected by the wild AcMNPV. AcMNPV without the $v$ cath gene was unable to cause the liquidization of the larvae (Liu et al., 2001).

This study revealed the interaction of HaNPV VP39 with the actin of the Hz-AM1 cells with the yeast two-hybrid system and the fluorescence microscopy technology. The transiently-expressed HaNPV VP39 could induce the actin cytoskeleton to aggregate sequentially - first, in cytoplasm, then in the nucleus. The cells became round after HaNPV VP39 was expressed, which may be due to the actin aggregation. However, the following still needs to be clarified: what is the function of this interaction in the virus infection? Are other viral proteins involved in the actin aggregation?

The work of cloning another important gene that is associated with the host liquidization (HaNPV v-cath) is ongoing. Hopefully, further work in this lab will reveal the liquidization mechanism of the Heliothis armigera larvae that is caused by HaNPV, and will lead to the construction of a recombinant HaNPV with an improved pest-killing effect.

Acknowledgments Special thanks to the China National Natural Science Foundation (CNNSF) for their financial support. (No. 39870041)

\section{References}

Ayres, M. D., Howard, S. C., Kuzio, J., Lopez-Ferber, M. and Possee, R. D. (1994) The complete DNA sequence of Autographa californica nuclear polyhedrosis virus. Virology 202, 586-605.

Bradley, T. J. and Satir, P. (1979) Evidence of microfilamentassociated mitochondrial movement; in Biological Recognition and Assembly, Eisenberg, D., Lake, J. and Fox, C. (eds.), pp. 129-139, Alan R. Liss, Inc., New York, New York.

Blissard, G. W. and Wenz, J. R. (1992) Baculovirus gp64 envelope glycoprotein is sufficient to mediate $\mathrm{pH}$-dependent membrane fusion. J. Virol. 66, 6829-6835.

Bjornson, R. M. and Rohrman, G. F. (1992) Nucleotide sequence of the p39-capsid gene region of the Lymantria dispar nuclear polyhedrosis virus. J. Gen. Virol. 73, 1505-1508.

Chariton, C. A. and Volkman, L. Z. (1991) Sequential rearrangement and nuclear polymerization of actin in Baculovirus-infected Spodoptera cells. J. Virol. 65, 1219-1227.

Chariton, C. A. and Volkman, L. Z. (1993) Penetration of Autographa californica nuclear polyhedrosis virus nucleocapsid into IPLB sf 21 cells induces actin cable formation. Virology 197, 245-254.

Chen, X., Ijkel, W. F., Tarchini, R., Sun, X., Sandbrink, H., Wang, H., Peters, S., Zuidema, D., Lankhorst, R. K., Vlak, J. M. and $\mathrm{Hu}, \mathrm{Z}$. (2001) The sequence of the Helicoverpa armigera single nucleocapsid nucleopolyhedrovirus genome. J. Gen. Virol. 82, 241-257.

Ciampor, F. (1988) The role of cytoskeleton and nuclear matrix in virus replication. Acta Virol. 32, 168-189.

Field, S. and Song, O. (1989) A novel genetic system to detect protein-protein interactions. Nature 340, 245-246.

Kachar, B. and Reese, T. S. (1988) The mechanism of cytoplasmic streaming in characaen algal cells: sliding of endoplasmic reticulum along actin filaments. J. Cell Biol. 106, 1545-1552.

Lorene, M. L., Jeffrey, M. S. and Volkman, L. Z. (1996) Actin binding and proteolysis by the baculovirus AcMNPV: the role of virion-associated V-CATH. Virology 216, 380-388.

Leikina, E., Onaran, H. O. and Zimmerberg, J. (1992) Acidic pH induces fusion of cells infected with baculovirus to form syncytia. FEBS Lett. 304, 221-224.

Liu, D. L., Sun, X. J., Qi, Y. P., Li X. F. and Zhu, Y. (1999) Cloning and expression of the $v p 39$ gene from Autographa californica nuclear polyhedrosis virus and its function. Chinese J. Biochem. Mol. Biol. 15, 340-343.

Liu, D. L., Xiao, H. Z., Qi, Y. P. and Yao L. G. (2001) Construction and function of the recombinant AcMNPV with two copies of v-cath gene. Chinese Sci. Bull. 46, 1288-1294.

Lorene, M. L. and Volkman, L. Z. (1998) Actin binding and nucleation by Autographa californica M nucleopolyhedrovirus. Virology 243, 167-177.

Ohkawa, T. and Volkman, L. E. (1999) Nuclear F-actin is required for AcMNPV nucleocapsid morphogenesis. Virology 264, 1-4.

Peng, H. Y., Li, X., Zhong S. M., Basil, M. A., Chen X. W. and Hu Z. H. (1998) Localization and cloning of the chitinase gene of Heliothis armigera single nucleocapsid nucleopolyhedrosis. Virologica Sinica 13, 139-143.

Qi B., Qi Y. P., Masuo, Y. and Liu, Q. Z. (2000) Isolation and characterization of a human apoptosis-inducing gene with yeast 
two-hybrid system. Science in China (series C) 43, 310-320.

Rourke, I. J. and East, P. D. (1997) Evidence for gene conversion between tandemly duplicated cytoplasmic actin genes of Helicoverpa armigera. J. Mol. Evol. 44, 167-177.

Stockem, W., Hoffmann, H. U. and Gruber, B. (1983) Dynamics of the cytoskeleton in Amoeba proteus.I. Redistribution of microinjected fluorescein-labeled actin during locomotion, immobilization and phagocytosis. Cell Tissue Res. 232, 79-96.

Stossel, T. P. (1984) Contribution of the structure of the cytoplasmic matrix. J. Cell Biol. 99, 15-21.

Taro, O. and Volkman, L. E. (1999) Nuclear F-Actin is required for AcMNPV nucleocapsid morphogenesis. Virology 264, 1-4. Tasaka, K., Akagi, M., Miyoshi, K. and Mio, M. (1988) Role of microfilaments in the exocytosis of rat peritoneal mast cells. Int. Arch. Allergy Appl. Immunol. 87, 213-221.

Thiem, S. M. and Miller, L. K. (1989) Identification, sequence, and transcriptional mapping of the major capsid protein of gene of the baculovirus Autographa californica multiple nuclear polyhedrosis virus. J. Virol. 63, 2008-2018.

Volkman, L. E. (1986) The $64 \mathrm{k}$ envelope protein of budded Autographa californica nuclear polyhedrosis virus. Curr. Top. Microbiol. Immunol. 131, 103-118. 